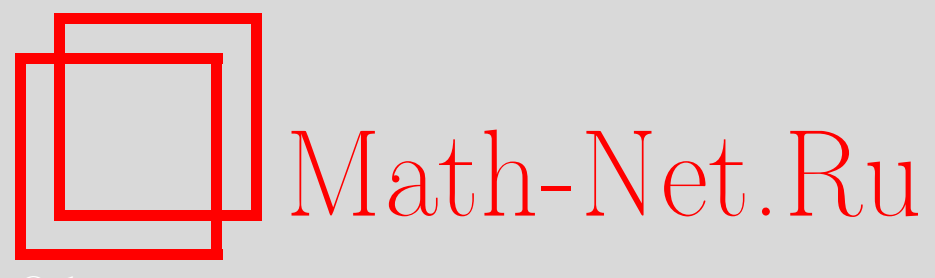

В. Г. Михайлов, Предельные теоремы для числа ненулевых решений одной системы случайных уравнений над полем GF(2), Дискрет. матем., 2000, том 12, выпуск $1,70-81$

DOI: https://doi.org/10.4213/dm318

Использование Общероссийского математического портала Math-Net.Ru подразумевает, что вы прочитали и согласны с пользовательским соглашением http://www.mathnet.ru/rus/agreement

Параметры загрузки:

IP: 34.229 .45 .116

26 апреля 2023 г., 15:16:54 


\title{
Предельные теоремы для числа ненулевых решений одной системы случайных уравнений над полем $\mathrm{GF}(2)$
}

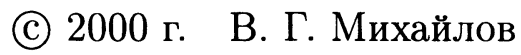

\begin{abstract}
Исследуются свойства числа $\nu$ ненулевых решений системы случайных уравнений, левые части которых являются произведениями выражений вида $a_{t 1} x_{1}+\ldots+a_{t n} x_{n}+a_{t}$ в поле $\mathrm{GF}(2)$ с независимыми равновероятными коэффициентами при переменных, а правые части равны нулю. Получены неравенства для факториальных моментов случайной величины $\nu$, а также необходимые и достаточные условия выполнения для $\nu$ предельной теоремы Пуассона.

Работа выполнена при поддержке Российского фонда фундаментальных исследований, проект 99-01-00012, и Совета по грантам Президента РФ и государственной поддержке ведущих научных школ, проект 96-15-96092.
\end{abstract}

Рассмотрим систему случайных нелинейных уравнений над полем GF(2)

$$
\prod_{i=1}^{m_{t}}\left(a_{t 1}^{i} x_{1}+\ldots+a_{t n}^{i} x_{n}+a_{t}^{i}\right)=0, \quad t=1, \ldots, T
$$

где $m_{t} \in\{1, \ldots, n\}$, а коэффициенты $a_{t j}^{i}$ являются независимыми случайными величинами, принимающими значения 0 и 1 с вероятностями $1 / 2$. Величины $a_{t}^{i}$ считаются заданными. Если при всех $t=1, \ldots, T$ выполнено равенство $a_{t}^{1} \ldots a_{t}^{m_{t}}=0$, то система (1) имеет нулевое решение. В остальных случаях его нет. Чтобы не загромождать изложение разбором этих двух случаев, мы будем изучать число $\nu$ ненулевых решений системы (1).

Введем обозначение $V^{n}$ для $n$-мерного линейного пространства над полем $\mathrm{GF}(2)$ и обозначение $(m)_{r}=m(m-1) \ldots(m-r+1)$. Положим

$$
\Lambda=\left(2^{n}-1\right) \prod_{t=1}^{T}\left(1-2^{-m_{t}}\right)
$$

Как будет показано ниже (см. равенство (19)), $\Lambda=\mathbf{E} \nu$. 
Теорема 1. При $n \geqslant r \geqslant 1$ выполнены неравенства

$$
\begin{aligned}
& \mathbf{E}(\nu)_{r} \geqslant \Lambda^{r} \prod_{s=0}^{r-1} \frac{2^{n}-2^{s}}{2^{n}-1} \\
& \mathbf{E}(\nu)_{r}<\Lambda^{r}+\left(\Lambda+2^{r-1}\right)^{r} \prod_{t=1}^{T}\left(1-\frac{2^{m_{t}}-2}{\left(2^{m_{t}}-1\right)^{2}}\right) .
\end{aligned}
$$

Введем обозначение

$$
\Delta\left(m_{1}, \ldots, m_{T}\right)=\sum_{t=1}^{T} \frac{2^{m_{t}}-2}{\left(2^{m_{t}}-1\right)^{2}} .
$$

Теорема 2. Пусть параметры п и Т стремятся $\kappa$ бесконечности, а параметры $m_{1}, \ldots, m_{T}$ меняются так, что $\Lambda \rightarrow \lambda, 0<\lambda<\infty, u$

$$
\Delta\left(m_{1}, \ldots, m_{T}\right) \rightarrow \infty
$$

Тогда равномерно по наборам свободнъх членов $\left\{a_{t}^{i}\right\}$

$$
\mathbf{E}(\nu)_{r} \rightarrow \lambda^{r}, \quad r=1,2, \ldots,
$$

а распределение случайной величины $\nu$ сходится $к$ распределению Пуассона с параметром $\lambda$ и эта сходимость равномерна по наборам свободных членов $\left\{a_{t}^{i}\right\}$.

Теорема 3. Пустъ параметры $n$ и $T$ стремятся $к$ бесконечности, а параметры $m_{1}, \ldots, m_{T}$ меняются таким образом, что выполнены условия

$$
\Lambda \rightarrow \infty, \quad \Lambda \leqslant \frac{n}{10}, \quad \Lambda^{2} \leqslant \frac{\Delta\left(m_{1}, \ldots, m_{T}\right)}{49 \ln 2} .
$$

Тогда функция распределения случайной величины $(\nu-\Lambda) \Lambda^{-1 / 2}$ сходится $x$ стандартной нормалъной функции распределения равномерно по наборам $\left\{a_{t}^{i}\right\}$.

Замечание 1. Из теорем 1, 2 и 3 вытекают аналогичные утверждения для системы вида (1) со случайными свободными членами $a_{t}^{i}$. В этом случае распределение свободных членов может быть произвольным, достаточно предполагать лишь независимость набора $\left\{a_{t}^{i}\right\}$ от набора случайных коэффициентов $\left\{a_{t j}^{i}\right\}$. Кстати, при равновероятном выборе набора $\left\{a_{t}^{i}\right\}$ из $2^{M}, M=m_{1}+\ldots+m_{T}$, возможных оказывается, что при условии (5) вероятность наличия нулевого решения стремится к нулю. Поэтому в условиях теоремы 2 имеет место предельная теолрема Пуассона с параметром $\lambda$ и для общего числа решений.

Замечание 2. Случай $m_{1}=\ldots=m_{T} \geqslant 2$ изучался в работе [1], где были получены утверждения, аналогичные теоремам 1,2 и 3 , и ряд иных результатов. В отличие от [1] в настоящей работе допускается ситуация, когда в систему (1) входят уравнения различных степеней (здесь под степенью уравнения понимается число сомножителей произведения в левой части (1), которое, разумеется, с некоторой вероятностью может отличаться от истинной степени случайного уравнения), в том числе и линейные уравнения. 
Замечание 3. Условие (5) теоремы 2 эквивалентно условию

$$
\sum_{t: m_{t} \geqslant 2} 2^{-m_{t}} \rightarrow \infty
$$

Если все $m_{t} \geqslant 2$, то условие (8) (а значит, и условие (5)) следует из условия $\Lambda \rightarrow \lambda$, $0<\lambda<\infty$. Поскольку $\Lambda=E \nu$, это означает, что условие (5) является в данном случае необходимым для сходимости моментов случайной величины $\nu$ к моментам предельного пуассоновского распределения.

Следующее утверждение показывает, что и в общем случае условие (5) необходимо для сходимости (6).

Теорема 4. Пусть при $n \rightarrow \infty$

$$
\mathbf{E}(\nu)_{r} \rightarrow \lambda^{r}, \quad r=1,3,4
$$

Тогда выполнено условие (5).

Описание основных задач теории систем случайных уравнений над конечными полями можно найти в книгах $[2,3]$ и работах $[4,5,6]$. Задача о числе решений системы случайных уравнений тесно связана с задачей о числе непокрытых точек при покрытии конечного множества его случайно выбираемыми подмножествами. Роль таких подмножеств играют дополнения к множествам решений отдельных уравнений системы (см. [7]).

Важным классом систем случайных уравнений являются заведомо совместные системы случайных уравнений. Асимптотические свойства числа решений ряда таких систем нелинейных уравнений изучалось в работах [8] и [9]. В работах [10-12] исследовались некоторые заведомо совместные системы нелинейных уравнений, в которых случайной была лишь правая часть. В этих работах в качестве предельных появлялись логарифмически пуассоновское и логарифмически нормальное распределения. В литературе отмечалось также, что в качестве предельного распределения для числа ложных решений заведомо совместных систем случайных булевых уравнений может выступать и пуассоновское распределение (см., например, [13]).

Перейдем к доказательствам.

Доказательство теоремы 1 во многом повторяет рассуждения работы [1]. Однако мы приводим его достаточно подробно, поскольку ряд промежуточных результатов потребуется при доказательстве теоремы 4.

Система уравнений (1) эквивалентна системе из $T m_{t}$-мерных включений (здесь и далее под включением понимается соотношение типа вектор принадлежит множеству)

$$
\left(\begin{array}{c}
a_{t 1}^{1} x_{1}+\ldots+a_{t n}^{1} x_{n} \\
\ldots \\
a_{t 1}^{m_{t}} x_{1}+\ldots+a_{t n}^{m_{t}} x_{n}
\end{array}\right) \in D\left(\begin{array}{c}
a_{t}^{1} \\
\ldots \\
a_{t}^{m_{t}}
\end{array}\right), \quad t=1, \ldots, T
$$

где множество $D(a), a \in V^{m_{t}}$, получается из множества $V^{m_{t}}$ выбрасыванием вектора $a+1^{m_{t}}$ (вектор $1^{m}$ имеет размерность $m$ и состоит из одних единиц, аналогичный смысл имеет обозначение $\left.0^{m}\right)$. 
Запишем систему включений (10) относительно вектора $x=\left(x_{1}, \ldots, x_{n}\right)$ (рассматриваемого как вектор-столбец) как одно включение

$$
A x \in B
$$

в пространстве размерности

$$
M=m_{1}+\ldots+m_{T}
$$

Для этого обозначим матрицу размера $m_{t} \times n$ случайных коэффициентов линейных форм из левой части включения (10) через $A_{t}$, а вектор-столбец в обозначении множества в правой части (10) через $a_{t}$. В качестве матрицы $A$ возьмем матрицу размера $M \times n$, полученную расположением друг под другом в естественном порядке матриц $A_{t}, t=1, \ldots, T$. В качестве множества $B$ возьмем множество

$$
B=D\left(a_{1}\right) \times \ldots \times D\left(a_{T}\right) .
$$

Заметим, что

$$
|B|=\prod_{t=1}^{T}\left(2^{m_{t}}-1\right) .
$$

При выбранных $A$ и $B$ система (10) теперь запишется в виде (11).

Сопоставим каждой паре значений

$$
(x, b), \quad x \in V^{n} \backslash\left\{0^{n}\right\}, \quad b \in B,
$$

случайную величину

$$
\eta_{x, b}=I\{A x=b\}
$$

Тогда

$$
\nu=\sum_{x \in V^{n} \backslash\left\{0^{n}\right\}} \sum_{b \in B} \eta_{x, b} .
$$

Из этого равенства вытекает следующее выражение для факториальных моментов случайной величины $\nu$ :

$$
\mathbf{E}(\nu)_{r}=\sum_{S} \mathbf{E} \eta_{x^{1}, b^{1}} \ldots \eta_{x^{r}, b^{r}}
$$

где

$$
S=\left\{\left(\left(x^{1}, b^{1}\right), \ldots,\left(x^{r}, b^{r}\right)\right) \in\left(\left(x \in V^{n} \backslash\left\{0^{n}\right\}\right) \times B\right)^{r}:\left(x^{i}, b^{i}\right) \neq\left(x^{j}, b^{j}\right), i \neq j\right\} .
$$

Лемма 1. Пусть $n \geqslant r \geqslant k \geqslant 1, x^{1}, \ldots, x^{r} \in V^{n} \backslash\left\{0^{n}\right\}, u \operatorname{rank}\left(x^{1}, \ldots, x^{r}\right)=k$. Тогда если для любых $i_{1}, \ldots, l_{s}, s \leqslant r$, выполнено неравенство

$$
\operatorname{rank}\left(b^{i_{1}}, \ldots, b^{i_{s}}\right) \leqslant \operatorname{rank}\left(x^{i_{1}}, \ldots, x^{i_{s}}\right),
$$

mo

$$
\mathbf{E} \eta_{x^{1}, b^{1}} \ldots \eta_{x^{r}, b^{r}}=\frac{1}{2^{k M}}
$$


если

$$
\operatorname{rank}\left(b^{i_{1}}, \ldots, b^{i_{s}}\right) \geqslant \operatorname{rank}\left(x^{i_{1}}, \ldots, x^{i_{s}}\right)
$$

для некоторых $i_{1}, \ldots, i_{s}, s \leqslant r$, mo

$$
E \eta_{x^{1}, b^{1}} \ldots \eta_{x^{r}, b^{r}}=0
$$

Доказательство леммы 1 достаточно прозрачно, и мы его опустим.

Из леммы 1 вытекает следующий результат, которым мы будем неоднократно пользоваться в дальнейшем.

Лемма 2. Пусть $x^{1}, \ldots, x^{r} \in V^{n} \backslash\left\{0^{n}\right\} u \operatorname{rank}\left(x^{1}, \ldots, x^{r}\right)=k$. Тогда при любъх $b^{1}, \ldots, b^{r} \in B$ выполнено неравенство

$$
\mathbf{P}\left\{\eta_{x^{1}, b^{1}}=\ldots=\eta_{x^{r}, b^{r}}=1\right\} \leqslant \frac{1}{2^{k M}}
$$

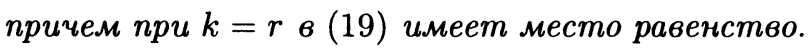

Непосредственно из леммы 1 следует равенство

$$
\mathbf{E}_{\nu}=\sum \eta_{x^{1}, b^{1}}=|B|\left|V^{n} \backslash\left\{0^{n}\right\}\right| 2^{-M}=\left(2^{n}-1\right) \prod_{t=1}^{T}\left(1-2^{-m_{t}}\right) .
$$

Рассмотрим старшие факториальные моменты (при $2 \leqslant r \leqslant n$ ). Введем обозначение

$$
S_{r, k}=\left\{\left(x^{1}, \ldots, x^{r}\right): x^{1}, \ldots, x^{r} \in V^{n} \backslash\left\{0^{n}\right\} \text { различны, } \operatorname{rank}\left(x^{1}, \ldots, x^{r}\right)=k\right\} .
$$

Очевидно, что $S_{r, 0}=S_{r, 1}=\varnothing ; r \geqslant 2, S_{r, 2}=\varnothing, r \geqslant 4$.

Заметим, что слагаемые в (14), для которых

$$
x^{i_{1}}+\ldots+x^{i_{s}}=0^{n}, \quad b^{i_{1}}+\ldots+b^{i_{s}} \neq 0^{M},
$$

равны нулю. Остальные группируем в суммы по $S_{r, k}, k=2, \ldots, r$, и получаем выражение

$$
\mathbf{E}(\nu)_{r}=\sum_{k=2}^{r} \sum_{\left(x^{1}, \ldots, x^{r}\right) \in S_{r, k}, b_{1}, \ldots, b^{r} \in B} \mathbf{E} \eta_{x^{1}, b^{1}} \ldots \eta_{x^{r}, b^{r}}
$$

Согласно лемме 2

$$
\sum_{\left(x^{1}, \ldots, x^{r}\right) \in S_{r, r}} \sum_{b_{1}, \ldots, b^{r} \in B} \mathbf{E} \eta_{x^{1}, b^{1}} \ldots \eta_{x^{r}, b^{r}}=\frac{|B|^{r}\left|S_{r, r}\right|}{2^{r M}}
$$

Поэтому с учетом равенства

$$
\left|S_{r, r}\right|=\prod_{s=0}^{r-1}\left(2^{n}-2^{s}\right)
$$


и формулы (12) получаем, что

$$
\sum_{\left(x^{1}, \ldots, x^{r}\right) \in S_{r, r}} \sum_{b_{1}, \ldots, b^{r} \in B} E \eta_{x^{1}, b^{1}} \ldots \eta_{x^{r}, b^{r}}=\Lambda^{r} \prod_{s=1}^{r-1} \frac{2^{n}-2^{s}}{2^{n}-1} .
$$

Из (20) и (21) следует (3).

Из (20) и (21) вытекает также равенство

$$
\mathbf{E}(\nu)_{2}=\frac{2^{n}-2}{2^{n}-1} \Lambda^{2} .
$$

Перейдем к построению верхней оценки. Каждому набору векторов $x^{1}, \ldots, x^{r}$ из $V^{n} \backslash\left\{0^{n}\right\}$ соответствует система $L_{x}\left(x^{1}, \ldots, x^{r}\right)$ однородных линейных уравнений в $V^{n}$ вида

$$
x^{i_{1}}+\ldots+x^{i_{s}}=0^{n},
$$

возможно пустая, описывающая все линейные соотношения между $x^{1}, \ldots, x^{r}$. Системе $L_{x}\left(x^{1}, \ldots, x^{r}\right)$ сопоставим идентичную систему $L_{b}\left(x^{1}, \ldots, x^{r}\right)$ из линейных уравнений относительно $b^{1}, \ldots, b^{r} \in B$. Обозначим через $B\left(x^{1}, \ldots, x^{r}\right)$ множество решений $\left(b^{1}, \ldots, b^{r}\right) \in B^{r}$ системы уравнений $L_{b}\left(x^{1}, \ldots, x^{r}\right)$.

Из (20) и леммы 2 следует оценка

$$
\mathbf{E}(\nu)_{r} \leqslant \sum_{k=2}^{r} \sum_{\left(x^{1}, \ldots, x^{r}\right) \in S_{r, k}} \frac{1}{2^{k M}}\left|B\left(x^{1}, \ldots, x^{r}\right)\right| .
$$

Обозначим через $C\left(r, d ; u_{1}, \ldots, u_{T}\right)$ число решений $\left(b^{1}, \ldots, b^{r}\right)$ уравнения

$$
b^{1}+\ldots+b^{r}=d, \quad b^{1}, \ldots, b^{r}, d \in B
$$

где

$$
B=B_{1} \times \ldots \times B_{T}, \quad B_{t}=V^{m_{t}} \backslash\left\{u_{t}\right\}, \quad u_{t} \in V^{m_{t}}, \quad t=1, \ldots, T .
$$

Лемма 3. Для любъх $u_{1}, \ldots, u_{T} u d$

$$
\begin{aligned}
\prod_{t=1}^{T}\left(2^{m_{t}}-1\right)\left(2^{m_{t}}-2\right) & \leqslant C\left(3, d ; u_{1}, \ldots, u_{T}\right) \\
& \leqslant \prod_{t=1}^{T}\left(\left(2^{m_{t}}-1\right)\left(2^{m_{t}}-2\right)+1\right) .
\end{aligned}
$$

Следствие 1. $\Pi p u r \geqslant 3 u \bumpeq ґ б ы x u_{1}, \ldots, u_{T} u d$

$$
C\left(r, d ; u_{1}, \ldots, u_{T}\right) \leqslant \prod_{t=1}^{T}\left(2^{m_{t}}-1\right)^{r-3}\left(\left(2^{m_{t}}-1\right)\left(2^{m_{t}}-2\right)+1\right) .
$$

Доказательство леммъ 3. При доказательстве можно считать, что $d=0$, так как общий случай сводится к этому частному случаю заменой ${b^{\prime}}^{i}=b^{i}+d, i=1,2,3$.

Сначала рассмотрим случай $T=1$, в котором уравнение (24) рассматривается над $B=V^{m} \backslash\{u\}$, где $u \in V^{m}$. 
Пусть $u=0^{m}$. Тогда решения уравнения (24) имеют вид $\left(b^{1}, b^{2}, b^{1}+b^{2}\right)$, где $b^{1}, b^{2} \neq 0^{m}, b^{1} \neq b^{2}$ (иначе $\left.b^{3}=0^{m}\right)$. Таких комбинаций имеется ровно $\left(2^{m}-1\right)\left(2^{m}-2\right)$ штук.

Пусть $u \neq 0^{m}$. Решениями являются

$$
\left(0^{m}, 0^{m}, 0^{m}\right), \quad\left(0^{m}, b, b\right), \quad\left(b, 0^{m}, b\right), \quad\left(b, b, 0^{m}\right)
$$

(где $\left.b \neq 0^{m}, u\right)$ и наборы вида $\left(b^{1}, b^{2}, b^{1}+b^{2}\right)$, где $b^{1}, b^{2} \neq 0^{m}, u$, причем $b^{1} \neq b^{2}, b^{2}+u$ (иначе $b^{3}=0^{m}$ или $b^{3}=u$ ). Итого, получаем

$$
1+3\left(2^{m}-2\right)+\left(2^{m}-2\right)\left(2^{m}-4\right)=\left(2^{m}-1\right)\left(2^{m}-2\right)+1
$$

решений.

При $T \geqslant 2$ число решений над $B=B_{1} \times \ldots \times B_{T}, B_{t}=V^{m_{t}} \backslash\left\{u_{t}\right\}$, равно произведению числа решений над $B_{t}, t=1, \ldots, T$. Отсюда получаем (25). Лемма 3 доказана.

Замечание 4. Пусть $m_{1}, \ldots, m_{T}$ и $u_{1}, \ldots, u_{T}$ таковы, что

$$
\left\{m_{t}=1\right\} \Longrightarrow\left\{u_{t}=1\right\}
$$

Тогда, согласно приведенным выше выкладкам,

$$
C\left(3, d ; u_{1}, \ldots, u_{T}\right) \geqslant \prod_{t=1}^{T}\left(\left(2^{m_{t}}-1\right)\left(2^{m_{t}}-2\right)+I\left\{m_{t}=1\right\}\right)
$$

Если же $m_{t}=1, u_{t}=0$ при некотором $t$, то

$$
C\left(3,0 ; u_{1}, \ldots, u_{T}\right)=0
$$

Замечание 5. Пусть среди уравнений системы (1) имеется уравнение

$$
a_{t 1}^{1} x_{1}+\ldots+a_{t n}^{1} x_{n}=1 .
$$

Тогда из равенств (28), (20) и (21) следует формула

$$
\mathbf{E}(\nu)_{3}=\frac{\left(2^{n}-2\right)\left(2^{n}-4\right)}{\left(2^{n}-1\right)^{2}} \Lambda^{3}
$$

Доказательство следствия 1. Фиксируем в уравнении (24) неизвестные $b^{4}, \ldots, b^{r}$, что можно сделать не более, чем $|B|^{r-3}$ способами. При этом получаем уравнение вида (24) с $r=3$, число решений которого оценивается сверху с помощью правого неравенства в (25). Отсюда и из (12) получаем оценку (26). Следствие доказано.

Лемма 4. Пусть $\operatorname{rank}\left(x^{1}, \ldots, x^{r}\right)=k u 2 \leqslant k \leqslant r-1$. Тогда

$$
\left|B\left(x^{1}, \ldots, x^{r}\right)\right| \leqslant \prod_{t=1}^{T}\left(2^{m_{t}}-1\right)^{k-2}\left(1-\frac{2^{m_{t}}-2}{\left(2^{m_{t}}-1\right)^{2}}\right) .
$$


Доказательство этой леммы аналогично доказательству леммы 4 из [1], и мы его опускаем.

Вернемся к неравенству (23). Подставив оценку (29) в (23) (для слагаемых с $k<r)$ и воспользовавшись равенством $(21)$, очевидными оценками

$$
\begin{gathered}
\left|B\left(x^{1}, \ldots, x^{r}\right)\right|<|B|^{r}, \\
\left|S_{r, k}\right| \leqslant C_{r}^{k}\left(2^{k}-1\right)^{r-k} \prod_{s=0}^{k-1}\left(2^{n}-2^{s}\right)
\end{gathered}
$$

и равенством (12), после несложных преобразований получим (4). Теорема 1 доказана.

Докажем теорему 2. Заметим, что из условия (5) следует, что

$$
\prod_{t=1}^{T}\left(1-\frac{2^{m_{t}}-2}{\left(2^{m_{t}}-1\right)^{2}}\right) \rightarrow 0
$$

Поэтому соотношение (6) следует из условия $\Lambda \rightarrow \lambda$ и неравенств (3) и (4) теоремы 1. Из него, в свою очередь, следует сходимость распределения. Обе сходимости равномерны по наборам $\left\{a_{t j}^{i}\right\}$. Теорема 2 доказана.

Доказательство теоремы 3 аналогично доказательству теоремы 3 из [1], и мы его опускаем.

При доказательстве теоремы 4 рассмотрим два частных случая.

Теорема 5. Пусть система (1) не содержит линейных уравнений вида

$$
a_{t 1}^{1} x_{1}+\ldots+a_{t n}^{1} x_{n}=1 .
$$

Тогда если при $n \rightarrow \infty$

$$
\mathbf{E}(\nu)_{r} \rightarrow \lambda^{r}, \quad r=1,3, \quad 0<\lambda<\infty
$$

то выполнено соотношение (5).

Теорема 6. Пусть система (1) содержит линейное уравнение вида (30). Тогда если при $n \rightarrow \infty$

$$
\mathbf{E}(\nu)_{r} \rightarrow \lambda^{r}, \quad r=1,4, \quad 0<\lambda<\infty,
$$

то выполнено соотношение (5).

Очевидно, что теорема 4 является прямым следствием теорем 5 и 6 .

Доказажем теорему 5. Из (20) и (21) следует равенство

$$
\mathbf{E}(\nu)_{3}=M_{3,2}+\frac{\left(2^{n}-2\right)\left(2^{n}-4\right)}{\left(2^{n}-1\right)^{2}} \Lambda^{3},
$$

где

$$
M_{3,2}=\sum_{\left(x^{1}, x^{2}, x^{3}\right) \in S_{3,2}} \sum_{b^{1}, b^{2}, b^{3} \in B} \mathrm{E} \eta_{x^{1}, b^{1}} \eta_{x^{2}, b^{2}} \eta_{x^{3}, b^{3}}
$$


Заметим, что

$$
\left.S_{3,2}=\left\{\left(x^{1}, x^{2}, x^{3}\right) \in\left(V^{n} \backslash\left\{0^{n}\right\}\right)^{3}: x^{i} \neq x^{j}(i \neq j), x^{1}+x^{2}+x^{3}=0\right)\right\} .
$$

Определим множество

$$
B_{3,2}=\left\{\left(b^{1}, b^{2}, b^{3}\right) \in B^{3}: b^{1}+b^{2}+b^{3}=0\right\} .
$$

Согласно второму утверждению леммы 1 суммирование по $B$ в выражении (34) для $M_{3,2}$ можно заменить на суммирование по множеству $B_{3,2}$. Поэтому в силу первого утверждения леммы 1 из (34) получаем равенство

$$
M_{3,2}=\frac{1}{2^{2 M}}\left|S_{3,2}\right|\left|B_{3,2}\right| .
$$

Заметим, что множество $B_{3,2}$ совпадает с множеством решений, изученным в лемме 3 , и для него в силу предположений теоремы справедлива оценка (27). Используя в (35) оценку (27), равенства (2) и

$$
\left|S_{3,2}\right|=\left(2^{n}-1\right)\left(2^{n}-2\right)
$$

получаем соотношения

$$
\begin{aligned}
M_{3,2} & \geqslant \frac{\left(2^{n}-1\right)\left(2^{n}-2\right)}{2^{2 M}} \prod_{t=1}^{T}\left(\left(2^{m_{t}}-1\right)\left(2^{m_{t}}-2\right)+I\left\{m_{t}=1\right\}\right) \\
& =\frac{2^{n}-2}{2^{n}-1} \Lambda^{2} \prod_{t=1}^{T}\left(1-\frac{2^{m_{t}}-1-I\left\{m_{t}=1\right\}}{\left(2^{m_{t}}-1\right)^{2}}\right) \\
& \geqslant \frac{2^{n}-2}{2^{n}-1} \Lambda^{2} \prod_{t=1}^{T}\left(1-\frac{3}{2} \frac{2^{m_{t}}-2}{\left(2^{m_{t}}-1\right)^{2}}\right)
\end{aligned}
$$

В силу (31), (33) и (36) при $n \rightarrow \infty$

$$
\prod_{t=1}^{T}\left(1-\frac{3}{2} \frac{\dot{2}^{m_{t}}-2}{\left(2^{m_{t}}-1\right)^{2}}\right) \rightarrow 0 .
$$

Последнее эквивалентно соотношению (5). Теорема 5 доказана.

Докажем теорему 6. Так как

$$
S_{4,0}=S_{4,1}=S_{4,2}=\varnothing,
$$

из (20) и (21) следует равенство

$$
\mathbf{E}(\nu)_{4}=M_{4,3}+\frac{\left(2^{n}-2\right)\left(2^{n}-4\right)\left(2^{n}-8\right)}{\left(2^{n}-1\right)^{3}} \Lambda^{4}
$$

где

$$
M_{4,3}=\sum_{\left(x^{1}, x^{2}, x^{3}, x^{4}\right) \in S_{4,3}} \sum_{b^{1}, b^{2}, b^{3}, b^{4} \in B} \mathrm{E} \eta_{x^{1}, b^{1}} \eta_{x^{2}, b^{2}} \eta_{x^{3}, b^{3}} \eta_{x^{4}, b^{4}}
$$


Пусть

$$
\begin{aligned}
& S_{4,3}^{(0)}=\left\{\left(x^{1}, x^{2}, x^{3}, x^{4}\right) \in S_{4,3}: x^{1}+x^{2}+x^{3}+x^{4}=0^{n}\right\}, \\
& S_{4,3}^{(1)}=\left\{\left(x^{1}, x^{2}, x^{3}, x^{4}\right) \in S_{4,3}: x^{2}+x^{3}+x^{4}=0^{n}\right\},
\end{aligned}
$$

a $S_{4,3}^{(2)}, S_{4,3}^{(3)}$ и $S_{4,3}^{(4)}$ определяются аналогично $S_{4,3}^{(1)}$ с заменой $x^{1}$ на $x^{2}, x^{3}$ и $x^{4}$ соответственно. Тогда, как нетрудно проверить, $S_{4,3}$ является объединением непересекающихся множеств $S_{4,3}^{(i)}, i=0, \ldots, 4$.

Определим также множества

$$
\begin{aligned}
& B_{4,3}^{(0)}=\left\{\left(b^{1}, b^{2}, b^{3}, b^{4}\right) \in B^{4}: b^{1}+b^{2}+b^{3}+b^{4}=0^{M}\right\}, \\
& B_{4,3}^{(1)}=\left\{\left(b^{1}, b^{2}, b^{3}, b^{4}\right) \in B^{4}: b^{2}+b^{3}+b^{4}=0^{M}\right\}
\end{aligned}
$$

a $B_{4,3}^{(2)}, B_{4,3}^{(3)}$ и $B_{4,3}^{(4)}$ определим аналогично $B_{4,3}^{(1)}$ с заменой $b^{1}$ на $b^{2}, b^{3}$ и $b^{4}$ соответственно. Заметим, что при наличии в системе (1) уравнения вида (30) множества $B_{4,3}^{(i)}$, $i=1,2,3,4$, пусты. Поэтому используя (38) и лемму 1 , получаем равенства

$$
\begin{aligned}
M_{4,3} & =\sum_{\left(x^{1}, x^{2}, x^{3}, x^{4}\right) \in S_{4,3}^{(0)}} \sum_{\left(b^{1}, b^{2}, b^{3}, b^{4}\right) \in B_{4,3}^{(0)}} \mathrm{E} \prod_{s=1}^{4} \eta_{x^{s}, b^{s}} \\
& =\frac{1}{2^{3 M}}\left|S_{4,3}^{(0)} \| B_{4,3}^{(0)}\right| .
\end{aligned}
$$

Лемма 5. При любом наборе $m_{1}, \ldots, m_{T}$ выполнено равенство

$$
\left|B_{4,3}^{(0)}\right|=\prod_{t=1}^{T}\left(\left(2^{m_{t}}-1\right)^{3}-\left(2^{m_{t}}-1\right)\left(2^{m_{t}}-2\right)\right) .
$$

Доказательство. Доказательство леммы 5 проводится по той же схеме, что и доказательство леммы 3. Только теперь речь идет о числе решений в, $B^{4}$ уравнения

$$
b^{1}+b^{2}+b^{3}+b^{4}=0^{M} .
$$

Сначала рассмотрим случай, в котором уравнение (41) рассматривается над $B=$ $V^{m} \backslash\{u\}$, где $u \in V^{m}$ (под $m$ и $u$ подразумеваются $m_{t}$ и $a_{t}+1^{m_{t}}$ соответственно).

Пусть $u=0^{m}$. Тогда решения уравнения (41) можно разбить на две группы. Решения из первой группы имеют вид $\left(b^{1}, b^{2}, b^{3}, b^{4}\right)$, где

$$
b^{1}=b^{2} \neq 0^{m} ; \quad b^{3}=b^{4} \neq 0^{m} .
$$

Таких комбинаций имеется ровно $\left(2^{m}-1\right)^{2}$ штук.

Решения из второй группы имеют вид $\left(b^{1}, b^{2}, b^{3}, b^{4}\right)$, где

$$
b^{1} \neq 0^{m} ; \quad b^{2} \neq 0^{m}, b^{1} ; \quad b^{3} \neq 0^{m}, b^{1}+b^{2} ; \quad b^{4}=b^{1}+b^{2}+b^{3} .
$$

Таких комбинаций имеется ровно $\left(2^{m}-1\right)\left(2^{m}-2\right)^{2}$ штук.

Всего получаем

$$
\left(2^{m}-1\right)^{2}+\left(2^{m}-1\right)\left(2^{m}-2\right)^{2}=\left(2^{m}-1\right)^{3}-\left(2^{m}-1\right)\left(2^{m}-2\right)
$$


решений.

Пусть $u \neq 0^{m}$. Тогда решения уравнения (41) можно разбить на три группы. Решения из первой группы имеют вид $\left(b^{1}, b^{2}, b^{3}, b^{4}\right)$, где

$$
b^{1}=b^{2} \neq u ; \quad b^{3}=b^{4} \neq u .
$$

Таких комбинаций имеется ровно $\left(2^{m}-1\right)^{2}$ штук.

Решения из второй группы имеют вид $\left(b^{1}, b^{2}, b^{3}, b^{4}\right)$, где

$$
b^{1}=0^{m} ; \quad b^{2} \neq u, 0^{m} ; \quad b^{3} \neq u, b^{2}+u ; \quad b^{4}=b^{2}+b^{3} .
$$

Таких комбинаций имеется ровно $\left(2^{m}-2\right)^{2}$ штук.

Решения из третьей группы имеют вид $\left(b^{1}, b^{2}, b^{3}, b^{4}\right)$, где

$$
b^{1} \neq u, 0^{m} ; \quad b^{2} \neq u, b^{1} ; \quad b^{3} \neq u, b^{1}+b^{2}+u ; \quad b^{4}=b^{1}+b^{2}+b^{3} .
$$

Таких комбинаций имеется ровно $\left(2^{m}-2\right)^{3}$ штук.

Получаем такое же, как и при $u=0^{m}$, суммарное число решений:

$$
\left(2^{m}-1\right)^{2}+\left(2^{m}-2\right)^{2}+\left(2^{m}-2\right)^{3}=\left(2^{m}-1\right)^{3}-\left(2^{m}-1\right)\left(2^{m}-2\right) .
$$

При $T \geqslant 2$ число решений над

$$
B=B_{1} \times \ldots \times B_{T}, \quad B_{t}=V^{m_{t}} \backslash\left\{u_{t}\right\}, \quad u_{t}=a_{t}+1^{m_{t}},
$$

равно произведению числа решений над $B_{t}, t=1, \ldots, T$. Отсюда получаем (40) в общем случае. Лемма 5 доказана.

Используя (39), (40), (2) и легко проверяемое равенство

$$
\left|S_{4,3}^{(0)}\right|=\left(2^{n}-1\right)\left(2^{n}-2\right)\left(2^{n}-4\right),
$$

получаем, что

$$
M_{4,3}=\frac{\left(2^{n}-2\right)\left(2^{n}-4\right)}{\left(2^{n}-1\right)^{2}} \Lambda^{3} \prod_{t=1}^{T}\left(1-\frac{2^{m_{t}}-2}{\left(2^{m_{t}}-1\right)^{2}}\right)
$$

Из (32), (37) и (42) вытекает соотношение (5). Теорема 6 доказана.

\section{Список литературы}

1. Михайлов В.Г., Предельные теоремы для числа ненулевых решений одной системы случайных уравнений над полем $\mathrm{GF}(2)$. Теория вероятностей и ее применения (1998) 43, №3, 598-606.

2. Коваленко И.Н., Левитская А.А., Савчук М.Н., Избранные задачи вероятностной комбинаторики. Наукова Думка, Киев, 1986.

3. Колчин В.Ф., Системы случайных уравнений. МИЭМ, Москва, 1988.

4. Балакин Г.В., Введение в теорию случайных систем уравнений. Труды по дискретной математике (1997) 1, 1-18. 
5. Балакин Г.В., Системы случайных систем уравнений над конечным полем. Трудъ по дискретной математике (1998) 2, 21-37.

6. Севастьянов Б.А., Чистяков В.П., О числе входных последовательностей, соответствующих выходной последовательности конечного автомата. Обозрение прикладной $u$ промышленной математики (1994) 1, №1, 96-107.

7. Сачков В.Н., Асимптотическое поведение числа $t$-минимальных покрытий. Дискретная математика (1993) 5, №1, 36-44.

8. Копытцев В.А., О распределении числа решений случайных заведомо совместных систем уравнений. Теория вероятностей и ее применения (1995) 40, №2, 430-437.

9. Михайлов В.Г., Предельные теоремы для случайного покрытия конечного множества и для числа решений системы случайных уравнений. Теория вероятностей u eе применения (1996) 41, №2, 272-283.

10. Копытцев В.А., О некоторых случайных заведомо совместных системах уравнений. Обозрение прикладной и промышленной математики (1994) 1, №1, 56-84.

11. Полин С.В., О случайных блужданиях на графе с отмеченным ребром. Обозрение прикладной и промышленной математики (1994) 1, №1, 85-92.

12. Михайлов В.Г., Асимптотическая нормальность логарифма числа пробразов выходной последовательности конечного автомата. Обозрение прикладной и промышленной математики (1994) 1, №1, 126-135.

13. Масол В.И., Теорема о предельном распределении числа ложных решений системы нелинейных случайных булевых уравнений. Теория вероятностей $и$ ее применения (1998) 43, №1, 41-56.

Статья поступила 24.12.1999. 